\title{
CLÍNICA E ACADEMIA ESCOLA DE EDUCAÇÃO FÍSICA: PRESCRIÇÃO DE EXERCÍCIOS FÍSICOS BASEADOS EM EVIDÊNCIAS CIENTIIFICAS
}

\author{
Marcos Roberto Queiroga \\ Universidade Estadual do Centro-Oeste do Paraná \\ queirogamr@hotmail.com \\ Sandra Aires Ferreira \\ Universidade Estadual do Centro-Oeste do Paraná \\ queirogasa@hotmail.com \\ Erica dos Santos Vaz \\ Universidade Estadual do Centro-Oeste do Paraná \\ erica.vaz@hotmail.com.br \\ Sara Carolina Scremin Souza \\ University of Ottawa \\ saracarolinassouza@hotmail.com
}

\author{
Lucas Eduardo Campos de Oliveira \\ Universidade Estadual do Centro-Oeste do Paraná \\ lucaseduardooliveira@hotmail.com \\ Natã Gomes de Lima Stavinski \\ Universidade Estadual do Centro-Oeste do Paraná \\ natavinski@hotmail.com \\ Daniel Zanardini Fernandes \\ Universidade Estadual do Centro-Oeste do Paraná \\ danizafe@gmail.com \\ Vinicius Müller Reis Weber \\ Universidade Estadual do Centro-Oeste do Paraná \\ viniciusweber1994@gmail.com \\ Danilo Fernandes da Silva \\ University of Ottawa \\ danilofernandesdasilva@hotmail.com
}

\begin{abstract}
Resumo
O objetivo deste trabalho foi descrever experiências e resultados vinculados ao projeto de extensão "Clínica e Academia Escola de Educação Física: prescrição de exercícios físicos baseados em evidências científicas”. O público atendido é constituído de pacientes portadores de doenças crônicas. A prescrição dos exercícios é planejada e ministrada por acadêmicos, pós-graduandos e docentes mediante informações individualizada do participante. O programa é elaborado considerando evidências científicas obtidas a partir de diretrizes e ensaios clínicos randomizados que respaldam a determinação da dose adequada de exercícios físicos individualizados. Resultados de 64 pacientes revelaram melhoras significativas na adiposidade abdominal, na força de preensão manual, na frequência cardíaca de repouso e na pressão arterial diastólica. Em relação à qualificação, observou-se envolvimento e interesse dos acadêmicos com programas de mestrado, de residência e com a prática profissional neste contexto, durante e após a graduação. A proposta extensionista tem potencial de desenvolvimento contínuo, aplicação prática e formação profissional, consolidando em importante papel das instituições de ensino superior no que diz respeito à consolidação da tríade acadêmica: ensino, pesquisa e extensão.

Palavras-chave: Doença Crônica. Exercício. Promoção da Saúde. Prática Clínica Baseada em Evidências. Relações Comunidade-Instituição.
\end{abstract}

\section{CLINIC AND ACADEMY SCHOOL OF PHYSICAL EDUCATION: PRESCRIPTION OF PHYSICAL EXERCISES BASED ON SCIENTIFIC EVIDENCE}

\begin{abstract}
The objective was to describe experiences and results linked to the extension project " Clinic and Academy School of Physical Education: prescription of physical exercises based on scientific evidence". The public served is composed of patients with chronic diseases. The prescription of the exercises is planned and administered by academics, postgraduates and teachers through individualized information of the participant. The program is based on scientific evidence obtained from guidelines and randomized clinical trials that support the determination of the adequate dose of individualized physical exercises. Results of 64 patients revealed significant improvements in abdominal adiposity, grip strength, resting heart rate and diastolic blood pressure. Regarding the qualification, it was observed the involvement and interest of the academics with masters programs, of residence and with professional practice in this context, during and after graduation. The extensionist proposal has the potential for continuous development, practical application and professional training, consolidating in an important role of the institutions of higher education with respect to the consolidation of the academic triad: teaching, research and extension.
\end{abstract}

Keywords: Chronic Disease. Exercise. Health Promotion. Evidence-Based Practice. Community-Institutional Relations. 
Clínica e Academia Escola de Educação Física: prescrição de exercícios físicos baseados em evidências científicas

\section{CLÍNICA Y ACADEMIA ESCUELA DE EDUCACIÓN FÍSICA: PRESCRIPCIÓN DE EJERCICIOS FÍSICOS BASADOS EN LA EVIDENCIA CIENTÍFICA}

\section{Resumen}

El objetivo fue describir experiencias y resultados vinculados al proyecto de extensión "Clínica y Academia Escuela de Educación Física: Prescripción de ejercicios físicos basados en la evidencia científica". El público atendido está compuesto por pacientes con enfermedades crónicas. La prescripción de los ejercicios es planeada y administrada por académicos, postgraduados y maestros a través de información individualizada del participante. El programa se basa en la evidencia científica obtenida de guías y ensayos clínicos aleatorios que apoyan la determinación de la dosis adecuada de ejercicios físicos individualizados. Los resultados de 64 pacientes revelaron mejoras significativas en la adiposidad abdominal, la fuerza de agarre manual, la frecuencia cardíaca en reposo y la presión arterial diastólica. En cuanto a la calificación, se observó la participación e interés de los académicos con programas de maestría, residencia y práctica profesional en este contexto, durante y después de la graduación. La propuesta extensionista tiene potencial para el desarrollo continuo, la aplicación práctica y la capacitación profesional, consolidándose en un papel importante de las instituciones de educación superior con respecto a la consolidación de la tríada académica: enseñanza, investigación y extensión.

Palabras clave: Enfermedad Crónica. Ejercicio. Promoción de la Salud. Práctica Clínica Basada en Evidencias. Relaciones Comunidad-Institución. 
Clínica e Academia Escola de Educação Física: prescrição de exercícios físicos baseados em evidências científicas

\section{INTRODUÇÃO}

O Colégio Americano de Medicina do Esporte (ACSM) e a Associação Médica Americana lançaram em 2007 a campanha o "Exercício é Remédio". O objetivo foi tornar a avaliação e a promoção da atividade física um padrão em cuidados clínicos e avançar na implementação de estratégias da atividade física baseada em evidência para promoção de saúde, prevenção e tratamento de doenças crônicas como a cardiovascular, o diabetes, o câncer, a hipertensão, a obesidade, a depressão e a osteoporose. No tratamento de doenças crônicas, o exercício físico complementa e pode substituir, em parte, intervenções médicas tradicionais, além de reduzir ou eliminar a necessidade de medicação (ACSM, 2018a).

Há evidências irrefutáveis da eficácia da atividade física regular na prevenção primária e secundária e no risco de mortalidade por várias doenças crônicas e condições de saúde (WARBURTON; NICOL; BREDIN, 2006; EDWARDS; LOPRINZI, 2016). Adicionalmente, os benefícios associados à prática de exercícios físicos podem ser tão poderosos quanto qualquer fármaco no tratamento destas enfermidades e, embora existam riscos potenciais, eles são minimizados com uma adequada abordagem e são superados em muito pelos benefícios (SALLIS et al., 2015; LOBELO; STOUTENBERG; HUTBER, 2014).

Para caracterizar o exercício físico como remédio é importante destacar a necessidade de se estabelecer dose, ou seja, qualidade e quantidade (WASFY; BAGGISH, 2016). Embora para algumas doenças ainda haja carência de informações que permitam estabelecer a dose adequada, Pedersen e Saltin (2015) fornecem uma base atualizada de evidências científicas para prescrever exercício físico no tratamento de 26 doenças.

Uma proposta fundamentada em evidências científicas com a finalidade de prescrever exercícios físicos como remédio e melhorar a qualificação profissional foi implantada no Curso de Educação Física da UNICENTRO-Guarapuava-PR-BR. Com esta missão, a Clínica e Academia Escola de Educação Física (CAEEF) presta serviços à comunidade, mediante implantação de um projeto de extensão que fornece orientação e prescrição de exercícios a portadores de doenças que podem ser agrupadas em psiquiátricas, neurológicas, metabólicas, cardiovasculares, pulmonares, desordens musculoesqueléticas e câncer (PEDERSEN; SALTIN, 2015). A formação teórico-prática de estudantes de Educação Física se fundamenta em um dos três ramos de atuação deste profissional no contexto da atenção primária à saúde, ou seja, as intervenções individualizadas (SILVA, 2016). Para garantir a dose de exercício os pacientes/casos são analisados e, levantamentos de guidelines, revisões sistemáticas e ensaios clínicos 
Clínica e Academia Escola de Educação Física: prescrição de exercícios físicos baseados em evidências científicas

randomizados fornecem recomendações e suporte para prescrição de exercícios físicos como alternativa terapêutica para as doenças (PEDERSEN; SALTIN, 2015; ACSM, 2018b).

O projeto "Clínica e Academia Escola de Educação Física: prescrição de exercícios físicos baseados em evidências científicas" é uma proposta extensionista de caráter educativo e científico que articula, amplia, desenvolve, realimenta e oportuniza a interação do aprendizado obtido em sala de aula com a prática, viabilizando a relação transformadora entre Universidade e sociedade. Nosso objetivo foi descrever experiências e resultados vinculados a este projeto tanto na prestação de serviços como na formação teórico/prática do futuro profissional de Educação Física na área de prescrição de exercícios baseados em evidência para populações especiais.

\section{MATERIAIS E MÉTODOS}

\section{Histórico e objetivos}

O projeto de extensão "Clínica e Academia Escola de Educação Física (CAEEF): prescrição de exercícios físicos baseados em evidências científicas" funciona no espaço com mesmo nome (CAEEF) é vinculada ao Departamento de Educação Física de Guarapuava da Universidade Estadual do Centro-Oeste do Paraná (UNICENTRO). Em junho de 2017 o projeto obteve financiamento externo por meio da Secretaria de Estado da Ciência e Tecnologia de Ensino Superior (SETI-PR) do Paraná (edital no 01/2016), programa de Extensão Universidade Sem Fronteiras (USF/SETI). O financiamento custeou bolsas com duração de 12 meses que permitiu a contratação de um profissional e quatro acadêmicos. Docentes e acadêmicos(as) de Educação Física, respectivamente, orientam e cumprem estágio pedagógico acadêmico obrigatório. Independente do financiamento externo, bolsistas e voluntários (Fisioterapia, Nutrição e Educação Física) de programas de extensão (quatro) e de iniciação científica (quatro) da UNICENTRO integram, permanentemente, a lista de colaboradores na clínica e no projeto, o que permite a manutenção da ação.

O projeto possui os seguintes objetivos: 1) Orientar e prescrever a prática individualizada de exercícios clínicos e terapêuticos baseados em evidências para o tratamento e recuperação de pacientes estáveis, porém, portadores de doenças psiquiátricas, neurológicas, metabólicas, cardiovasculares, pulmonares, desordens musculoesqueléticas e câncer, encaminhados por profissionais das áreas de saúde e; 2) atualizar e qualificar o profissional de Educação Física para atuar neste segmento. 
Clínica e Academia Escola de Educação Física: prescrição de exercícios físicos baseados em evidências científicas

\section{Público-alvo}

São elegíveis a participar das atividades de extensão, crianças, adolescentes, adultos e idosos estáveis para sinais (i.e. medicados e liberados para prática de atividades físicas, pressão arterial, glicemia sanguínea, dor aguda), encaminhados por profissionais do Sistema Único de Saúde, clínicas médicas do município e prestadoras de serviços da UNICENTRO (Clínicas de Fisioterapia, Órtese e Prótese, Farmácia Escola e de Nutrição, além dos programas de residência multiprofissional em saúde da família e de residência em clínica médica). Também são encaminhados pacientes de clínicas particulares bem como pacientes que souberam das atividades na Clínica por meio de amigos ou divulgação. O modelo lógico do funcionamento das atividades no projeto pode ser visualizado na Figura 1.

Considerando o período de funcionamento do projeto (junho de 2017 até o momento) foram atendidos aproximadamente 140 pacientes. Contudo, é interessante destacar que a grande maioria dos pacientes, em função da particularidade da condição de saúde, não são submetidos a todas as rotinas de avaliação (pacientes com lombalgia, disfunções pulmonares, lesão de menisco, hipertensão, portadores de duas ou mais patologias). Pacientes com multimorbidades, comumente são tratados como "estudo de caso". Para estes, o tipo de exercício prescrito não segue uma orientação tradicional e pode enfatizar, em maior proporção (parte principal - Quadro 1), exercícios de flexibilidade, neuromuscular ou resistido. Neste sentido, 64 pacientes, a maioria obesos que realizaram, predominantemente, exercícios aeróbios, foram incluídos na análise deste estudo.

\section{Triagem inicial}

Os pacientes encaminhados por equipe de saúde, geralmente trazem consigo exames e descrições sobre suas condições e necessidades. Todos são recebidos por profissionais ou docentes de Educação Física que apresentam a proposta e realizam uma entrevista para identificar e reunir informações a respeito do histórico de saúde (anamnese) e respondem a três questionários (Figura 1). Em seguida, e de acordo com a condição de saúde, são submetidos a testes e medidas para avaliação da aptidão morfofuncional, objetiva e subjetiva. São liberados para o ingresso imediato os pacientes que possuem encaminhamento e não apresentam restrições médicas graves para a realização de atividades físicas cotidianas, tais como tarefas domésticas, atividades de lazer e de tempo livre, caminhadas e outras similares consideradas atividades de intensidade leve (30 a 39\% $\mathrm{FC}_{\text {res }}$ ou $\mathrm{VO}_{2 \text { res }}$ ) a moderada (40 a $59 \% \mathrm{FC}_{\text {res }}$ ou $\mathrm{VO}_{2 \text { res }}$ ) (ACSM, 2018b). Além disso, caso nesta fase sejam reveladas restrições potencialmente graves para a realização de atividades físicas de intensidade moderada ou que o paciente não tenha sido 
Clínica e Academia Escola de Educação Física: prescrição de exercícios físicos baseados em evidências científicas

encaminhado com a descrição dos diagnósticos de patologias, ele é conduzido a um médico residente na instituição, ao médico da unidade de saúde do bairro ou a outro de preferência do paciente para que possa liberá-lo para a prática de exercícios.

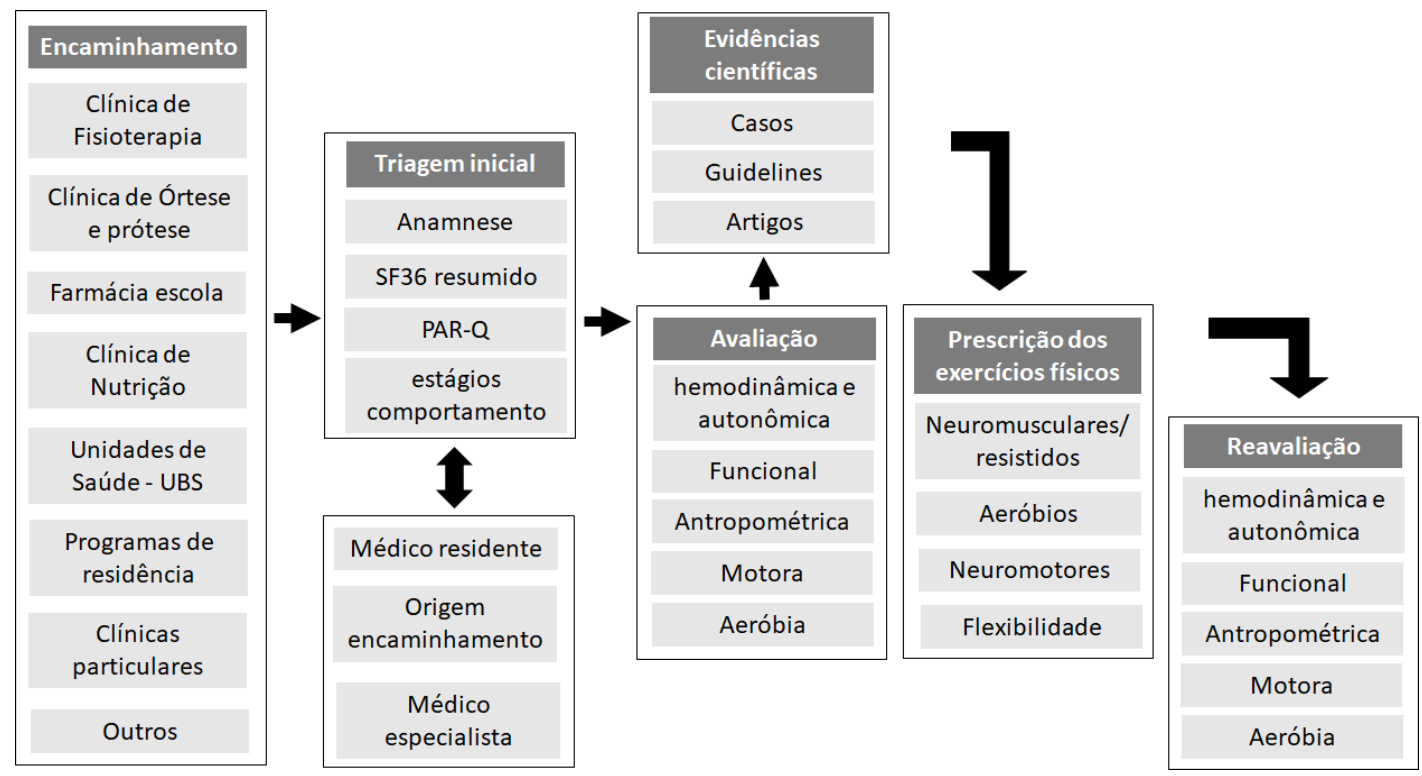

Figura 1. Modelo lógico de funcionamento das atividades do projeto de extensão na Clínica e Academia Escola de Educação Física de Guarapuava-PR

\section{Prática e prescrição de exercícios físicos baseado em evidências}

As atividades são oferecidas aos pacientes com frequência de duas vezes por semana $\left(2^{\mathrm{a}} \mathrm{a}\right.$ $5^{\text {a }}$ feira). A intensidade das atividades aeróbias é controlada a partir de um percentual da frequência cardíaca de reserva $\left(\mathrm{FC}_{\text {res }}\right)$. Além da $\mathrm{FC}$, os pacientes são questionados e relatam a percepção subjetiva de esforço (PSE) por meio de uma escala de 0 a 10 pontos (BORG, 2000) confeccionada em lona $(1,00$ x 1,20 m) e fixada em local visível na CAEEF. Cada paciente, antes do início da sessão, recebe um frequencímetro para monitoramento da FC. As sessões possuem duração de 60 minutos com atividades de preparação (parte inicial), parte principal e parte final (volta à calma), distribuídos em cada sessão e variando de duração conforme progressão no programa (Quadro 1).

Após a realização da triagem inicial e das avaliações, os casos são analisados pelos profissionais, acadêmicos, pós-graduandos e docentes, com a finalidade de planejar o programa de orientação e prescrição dos exercícios físicos de forma individualizada fundamentado nas características pessoais e nas informações obtidas. Uma consulta ao manual do ACSM (2018b) é realizada para identificar as recomendações para os quatro tipos de exercícios físicos, ou seja, o aeróbio, o neuromuscular (resistidos), o neuromotor e a flexibilidade. A orientação dos exercícios segue o acrônimo dos princípios de prescrição, denominado FITT-VP (frequência, intensidade, 
Clínica e Academia Escola de Educação Física: prescrição de exercícios físicos baseados em evidências científicas

tempo, tipo - volume, progressão) e é administrado de forma pragmática, ou seja, associando as condições de atuação [condições reais] (e.g., materiais disponíveis, horários de intervenção, experiência prévias com atividades físicas) com as recomendações científicas [condições ideais]. Também é consultado o estudo de Pedersen e Saltin (2015) para compreender as recomendações específicas de exercícios físicos para doenças que possam ser comuns ao paciente, juntamente com levantamentos e análises de estudos com casos semelhantes na literatura (paciente e intervenção), adotando a estratégia PICO (SANTOS; PIMENTA; NOBRE, 2007) para a busca de evidências científicas disponíveis em forma de revisões sistemáticas, com ou sem metanálise, e ensaios clínicos randomizados.

Quadro 1. Modelo geral de planejamento e orientação do programa de exercícios físicos (dose) implantado na Clínica e Academia Escola de Educação Física de Guarapuava-PR

\begin{tabular}{|c|c|c|c|c|c|c|}
\hline & $\mathbf{F}$ & I & $\mathbf{T}$ & $\mathbf{T}$ & $\mathbf{V}$ & $\mathbf{P}$ \\
\hline \multicolumn{7}{|c|}{ Avaliação pré intervenção } \\
\hline \multirow{4}{*}{$\begin{array}{c}\text { Mesociclo } 1 \\
1 \text { a } 30 \text { dias }\end{array}$} & \multirow{4}{*}{2} & \multirow{4}{*}{$\begin{array}{c}40-59 \% \\
\text { FC }_{\text {reserva }} \\
2 \text { a } 4 \text { PSE }\end{array}$} & $20-60 \mathrm{~min}$ & Aeróbio, & $40-120$ & \multirow{4}{*}{ ITT } \\
\hline & & & PI: até 25 min & Neuromuscular, & PI: até $50 \mathrm{~min}$ & \\
\hline & & & PP: até $25 \mathrm{~min}$ & Neuromotor, & PP: até $50 \mathrm{~min}$ & \\
\hline & & & PF: até $10 \mathrm{~min}$ & Flexibilidade & PF: até $20 \mathrm{~min}$ & \\
\hline \multirow{4}{*}{$\begin{array}{l}\text { Mesociclo } 2 \\
30 \text { a } 60 \text { dias }\end{array}$} & \multirow{4}{*}{2} & \multirow{4}{*}{$\begin{array}{l}\text { 60-80\% } \\
\text { FC }_{\text {reserva }} \\
4 \text { a } 6 \text { PSE }\end{array}$} & $60 \mathrm{~min}$ & Aeróbio, & 120 & \multirow{4}{*}{ ITT } \\
\hline & & & PI: até $20 \mathrm{~min}$ & Neuromuscular, & PI: até $40 \mathrm{~min}$ & \\
\hline & & & PP: até $30 \mathrm{~min}$ & Neuromotor, & PP: até $60 \mathrm{~min}$ & \\
\hline & & & PF: até $10 \mathrm{~min}$ & Flexibilidade & PF: até $20 \mathrm{~min}$ & \\
\hline \multirow{4}{*}{$\begin{array}{l}\text { Mesociclo } 3 \\
60 \text { a } 90 \text { dias }\end{array}$} & \multirow{4}{*}{2} & \multirow{4}{*}{$\begin{array}{l}70-89 \% \\
\text { FC }_{\text {reserva }} \\
\geq 6 \mathrm{PSE}\end{array}$} & $60 \mathrm{~min}$ & Aeróbio, & 120 & \multirow{4}{*}{ I'T'T } \\
\hline & & & PI: até $20 \mathrm{~min}$ & Neuromuscular, & PI: até $40 \mathrm{~min}$ & \\
\hline & & & PP: até $30 \mathrm{~min}$ & Neuromotor, & PP: até $60 \mathrm{~min}$ & \\
\hline & & & PF: até $10 \mathrm{~min}$ & Flexibilidade & PF: até $20 \mathrm{~min}$ & \\
\hline
\end{tabular}

\section{Avaliação pós intervenção}

*PI; parte inicial; PP: parte principal; PF: parte final; PSE: percepção subjetiva de esforço (Borg $\left.{ }^{10}\right)$; F: frequência; I: Intensidade; T: tempo/duração; T: Tipo/modo; V: Volume (F*T); P: Progressão (intensidade, tempo e tipo de exercício - complexidade de execução). Tipos/modos de exercícios: aeróbios (caminhada, circuito/estações), neuromusculares (exercícios resistidos estáticos e dinâmicos, com e sem oclusão vascular), neuromotores (exercícios corretivos de estabilidade, equilíbrio, coordenação, agilidade, propriocepção), flexibilidade (liberação miofascial, mobilidade, corretivos de estabilidade, alongamentos estáticos e dinâmicos); ACSM (2018b).

\section{Análise dos dados}

Informações de 64 pacientes (16 homens e 48 mulheres) foram comparadas antes e após um período de 90 dias de intervenção. A média e $( \pm)$ desvio padrão foram empregados para a descrição dos dados e o teste $\mathrm{t}$ para amostra dependentes foi utilizado para comparar os momentos pré e pós-intervenção, com nível de significância de $\mathrm{p}<0,05$. 
Clínica e Academia Escola de Educação Física: prescrição de exercícios físicos baseados em evidências científicas

\section{RESULTADOS E DISCUSSÃO}

Embora os resultados das avaliações obtidos antes e após a participação dos pacientes no programa individualizado de exercícios na CAEEF sejam promissores (redução da gordura abdominal, da frequência cardíaca em repouso e da pressão arterial diastólica e, aumento da força muscular) (Tabela 1), é preciso enfatizar que as análises no contexto de estudos de caso são clinicamente mais relevantes. Há enorme dificuldade em parear os pacientes atendidos, uma vez que apresentam faixa etária, características físicas e de saúde muito peculiares. É comum o atendimento de pacientes com duas ou mais patologias agregadas, que pode ser simplificado com os seguintes casos: paciente que apresenta diabetes tipo 1, depressão, síndrome metabólica e labirintite (VAZ et al., 2018), paciente diagnosticado com ataxia cerebral e obesidade, paciente portador de distúrbio pulmonar obstrutivo crônico (DPOC), lombalgia, hipertensão, obesidade, etc.

Tabela 1. Informações de pacientes antes e após três meses de atendimento no Projeto de extensão

\begin{tabular}{|c|c|c|c|c|}
\hline \multirow{2}{*}{ Demografia } & $\operatorname{Sexo}(\mathrm{n} \hat{\sigma} / q)$ & \multicolumn{2}{|c|}{$16 / 48$} & \\
\hline & Idade (anos) $(\widehat{\sigma} / q)$ & \multicolumn{2}{|c|}{ 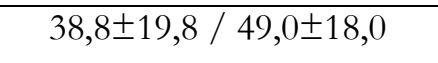 } & \\
\hline & Variáveis & Pré & Pós & $\mathbf{P *}$ \\
\hline \multirow{4}{*}{ Antropometria } & Massa corporal (kg) & $81,6 \pm 17,1$ & $80,8 \pm 16,8$ & 0,132 \\
\hline & Estatura $(\mathrm{cm})$ & \multicolumn{2}{|c|}{$160,0 \pm 0,9$} & - \\
\hline & $\mathrm{CC}(\mathrm{cm})$ & $97,1 \pm 16,4$ & $92,30 \pm 14,0$ & 0,001 \\
\hline & IMC $\left(\mathrm{kg} / \mathrm{m}^{2}\right)$ & $31,6 \pm 6,2$ & $29,5 \pm 9,7$ & 0,105 \\
\hline \multirow{4}{*}{ Testes motores } & Sentar-e-levantar (seg) & $10,0 \pm 1,9$ & $9,6 \pm 1,6$ & 0,124 \\
\hline & Flexibilidade $(\mathrm{cm})$ & $23,7 \pm 10,5$ & $24,2 \pm 11$ & 0,424 \\
\hline & PMD (kg) & $33,2 \pm 10,5$ & $37,4 \pm 8,1$ & 0,006 \\
\hline & PME (kg) & $32,0 \pm 10,2$ & $34,7 \pm 8,1$ & 0,045 \\
\hline \multirow{2}{*}{$\begin{array}{c}\text { Composição } \\
\text { corporal }\end{array}$} & Massa magra (\%) & $58,7 \pm 10,2$ & $59,6 \pm 9,8$ & 0,201 \\
\hline & $\%$ Gordura & $41,1 \pm 10,2$ & $40,9 \pm 10,6$ & 0,642 \\
\hline \multirow{5}{*}{$\begin{array}{c}\text { Funcional } \\
\text { hemodinâmico e } \\
\text { aeróbio }\end{array}$} & Frequência cardíaca (rep) & $84,0 \pm 12,1$ & $78,7 \pm 14,0$ & 0,002 \\
\hline & PAS (mmHg) & $128,4 \pm 11,4$ & $126,6 \pm 11,3$ & 0,156 \\
\hline & PAD (mmHg) & $80,2 \pm 7,9$ & $78,3 \pm 6,6$ & 0,009 \\
\hline & Capacidade vital (L) & $3,2 \pm 7,9$ & $3,3 \pm 9,0$ & 0,113 \\
\hline & $\mathrm{VO}_{2}$ pico $\left(\mathrm{mL} \cdot \mathrm{kg} \cdot \mathrm{min}^{-1}\right)$ & $29,3 \pm 6,9$ & $32,0 \pm 5,9$ & 0,093 \\
\hline
\end{tabular}

*Teste t para amostras dependentes; Média \pm Desvio padrão; CC: circunferência da cintura; CQ: circunferência do quadril; IMC: índice de massa corporal; PMD/PME: preensão manual direita e esquerda; PAS: pressão arterial sistólica; PAD: pressão arterial diastólica 
Clínica e Academia Escola de Educação Física: prescrição de exercícios físicos baseados em evidências científicas

Essas características exigem formas de planejamento (dose) e condução do programa de exercícios (FITT-VP), bem como a comparação e análise de resultados, quase exclusivamente, para o mesmo paciente. Esta situação tem norteado os docentes, profissionais e acadêmicos envolvidos com a clínica e com o projeto, a propor como disseminação dos resultados, a apresentação de estudos de casos, que certamente poderão ser consultados em outras oportunidades.

Contudo, o objetivo deste estudo foi o de apresentar experiências práticas/teóricas acumuladas neste período de funcionamento do projeto, aproximadamente dois anos, na prestação de serviços e na formação teórico-prática do profissional de Educação Física na área de prescrição de exercícios baseados em evidência científica para populações especiais. Este período nos permitiu destacar algumas questões inerentes ao modelo de funcionamento da Clínica e do projeto (Figura 1; Tabela 1) no que se refere à interdisciplinaridade na extensão e na pesquisa, a formação acadêmica continuada (alunos/profissionais), o desenvolvimento de hábitos em realizar buscas de referenciais científicos para a prescrição de exercícios físicos e a aquisição de prática clínica. Este cenário teórico-prático concebido no ambiente da CAEEF, fornece autoconfiança ao acadêmico/profissional para estabelecer doses adequadas de exercícios e garante o cuidado aos pacientes atendidos.

O papel do profissional de saúde, na atenção primária, requer um modelo de atuação em equipe que é pouco explorado em cursos de graduação em saúde (CARÁCIO et al., 2014). Discutir este modelo vai além do escopo deste trabalho, porém, destaca-se que será a realidade destes profissionais na atenção primária (equipes multiprofissionais). A participação do acadêmico de Educação Física na CAEEF tem possibilitado a interdisciplinaridade por meio de projetos de extensão, pesquisa e grupos de estudos, com trocas de experiências e informações entre docentes e graduandos das cinco áreas da saúde da instituição (Fisioterapia, Farmácia, Enfermagem, Nutrição e Educação Física). Parte dos acadêmicos (estagiários, voluntários/bolsistas, de extensão e pesquisa) que vivenciaram o modelo de funcionamento da CAEEF e do projeto de extensão foram selecionados em programas de Mestrado (3), em programas de Residência (2) ou encontram-se atuando profissionalmente neste segmento (8) em clínicas, academias e treinamento personalizado.

Quanto à formação e atuação do profissional, especificamente na área de prescrição e orientação de exercícios físicos para populações especiais, notamos uma lacuna entre um paciente que recebe alta de um serviço de saúde (especialidades médicas, indicações médicas, fisioterapia, nutricionista) e o profissional de Educação Física que deveria orientar um programa de exercícios para dar continuidade ao seu tratamento usando ferramentas técnicas e científicas (e.g., FITTT-VP, 
Clínica e Academia Escola de Educação Física: prescrição de exercícios físicos baseados em evidências científicas

Ensaios Clínicos Randomizados, guidelines). Um aspecto determinante nesta formação é a necessidade de permitir o contato direto com os pacientes, seja na graduação ou na pósgraduação. Neste sentido, a CAEEF e o projeto têm oportunizado uma intensa proximidade entre acadêmicos e pacientes além de requerer a descrição de cada caso com as fontes de evidência que recomendam os exercícios físicos (PEDERSEN; SALTIN, 2015; ACSM, 2018b).

Após o paciente ser submetido a rotina de avaliação, suas informações são utilizadas para o levantamento de evidências científicas, por meio de estratégias de busca, com a finalidade de elaborar um programa com a dose de exercícios mais próxima possível das recomendações. Além de consultas realizadas em diretrizes que sugerem a prática de exercícios para enfermidades mais comuns (e.g., hipertensão, diabetes) (DIRETRIZES DA SOCIEDADE BRASILEIRA DE DIABETES, 2017; SOCIEDADE BRASILEIRA DE CARDIOLOGIA, 2016), são investigadas informações em estudos de revisão de revisões de literatura (PEDERSEN; SALTIN, 2015) e, caso a dose de exercícios para alguma patologia não seja determinada nestas primeiras pesquisas, buscas a partir da estratégia PICO são efetuadas nas principais bases de dados (SANTOS; PIMENTA; NOBRE, 2007). Não são raras as necessidades deste tipo de busca, uma vez que parte das diretrizes e revisões não especificam a quantidade e qualidade de exercícios para todos os tipos de enfermidades que demandam os atendimentos na CAEEF. Uma vez em posse de informações que subsidiem a organização dos componentes da prescrição de exercícios (FITTVP) o próximo passo é a escolha dos tipos de atividades. Neste momento, a parte principal do programa muitas vezes é ocupada com a realização de um tipo de exercício (aeróbio, neuromotor, neuromuscular, flexibilidade) que não segue a prescrição tradicional (e.g.; recomendações generalizadas de atividades físicas sem considerar especificidade da doença ou doenças).

Ressalta-se a relevância das atividades de implementação de evidências científicas em instituições que trabalham na área de saúde e a possibilidade de aplicação no contexto das ações individuais/domiciliares (SILVA, 2016) que fazem parte da rotina de profissionais de Educação Física que atuam no serviço público e privado. Destacamos a possibilidade de fornecer, por meio das vivências neste projeto, uma "ponte" entre a teoria e a prática profissional. Esta ação tem ganhado destaque em diferentes áreas da saúde com a denominação "prática baseada em evidências" (científicas) (PEDERSEN; SALTIN, 2015; KENT et al., 2018). Neste contexto, os acadêmicos envolvidos têm observado que esta "ponte" não é diferente para o profissional de Educação Física, que precisa possuir capacidade para se utilizar do exercício físico "baseado em evidências" como ferramenta de trabalho no tratamento de doenças crônicas.

A proposta desenvolvida na CAEEF e revelada no projeto de extensão tem potencial de desenvolvimento contínuo, aplicação prática e formação profissional, consolidando em 
Clínica e Academia Escola de Educação Física: prescrição de exercícios físicos baseados em evidências científicas

importante papel das instituições de ensino superior no que diz respeito à consolidação da tríade acadêmica: ensino, pesquisa e extensão.

\section{CONSIDERAÇÕES FINAIS}

A demanda por atendimentos clínicos individualizados relacionados a doenças crônicas tem aumentado e o papel do exercício físico como remédio tem se destacado. Evidências científicas respaldam recomendações de doses adequadas de exercícios físicos para diversos tipos de doenças crônicas e condições de saúde. Para o futuro profissional de Educação Física é preciso o desenvolvimento de habilidades práticas que são negligenciadas ou pouco exploradas nas instituições de formação, porém, de fundamental importância para atuar com populações especiais. A CAEEF e o projeto de extensão em questão têm desempenhado importante função tanto na prestação de serviços de qualidade a comunidade quanto na formação de alunos de graduação e pós-graduação em Educação Física no contexto interdisciplinar, acadêmico e profissional.

\section{AGRADECIMENTOS}

Agradecemos à Fundação Araucária pelo recurso concedido por meio do Programa de Extensão Universidade sem Fronteiras (USF/SETI-PR).

\section{REFERÊNCIAS}

AMERICAN COLLEGE OF SPORTS MEDICINE (ACSM). What is Exercise is Medicine ${ }^{\circledR}$. In: American College of Sports Medicine. All rights reserved worldwide, 2018a. Disponível em: https://www.exerciseismedicine.org/support_page.php/about-eim5/

AMERICAN COLLEGE OF SPORTS MEDICINE. Diretrizes do ACSM para os testes de esforço e sua prescrição. Rio de Janeiro: Guanabara Koogan, 2018b.

BORG G. Escalas de Borg para a dor e esforço percebido. São Paulo: Manole, 2000.

CARÁCIO, F.C.C.; CONTERNO, L.O.; OLIVEIRA, M.A.C.; OLIVEIRA, A.C.H.; MARIN, M.J.S.; BRACCIALLI, L.A.D. A experiência de uma instituição pública na formação do profissional de saúde para atuação em atenção primária. Ciência \& Saúde Coletiva, v. 19, n. 7, p. 2133-2142, 2014.

DIRETRIZES DA SOCIEDADE BRASILEIRA DE DIABETES 2017-2018 / Organização José Egídio Paulo de Oliveira, Renan Magalhães Montenegro Junior, Sérgio Vencio. São Paulo: Ed. Clannad, 2017. 
Clínica e Academia Escola de Educação Física: prescrição de exercícios físicos baseados em evidências científicas

EDWARDS, M.K.; LOPRINZI, P.D. All-cause mortality risk as a function of sedentary behavior, moderate-to-vigorous physical activity and cardiorespiratory fitness. Phys Sportsmed, v. 44, n. 3, p. 223-30, 2016.

KENT, P.; O'SULLIVAN, P.B.; KEATING, J.; SLADE, S.C. Evidence-based exercise prescription is facilitated by the Consensus on Exercise Reporting Template (CERT). Br J Sports Med., v. 52, n. 3, p.147-148, 2018.

LOBELO, F.; STOUTENBERG, M.; HUTBER, A. The Exercise is Medicine Global Health Initiative: a 2014 update. Br J Sports Med., v. 48, n. 22, p. 1627-33, 2014.

PEDERSEN, B.K.; SALTIN, B. Exercise as medicine - evidence for prescribing exercise as therapy in 26 different chronic diseases. Scand J Med Sci Sports.v. 25 (Suppl 3), p. 1-72, 2015.

SALLIS, R.; FRANKLIN, B.; JOY, L.; ROSS, R.; SABGIR, D.; STONE, J. Strategies for promoting physical activity in clinical practice. Prog Cardiovasc Dis., v. 57, n. 4, p. 375-86, 2015.

SANTOS, C.M.C.; PIMENTA, C.A.M.; NOBRE, M.R.C. A estratégia pico para a construção da pergunta de pesquisa e busca de evidências. Rev Latino-Am Enfermagem, v. 15, n. 3, p. 508-511, 2007.

SILVA PSC. Núcleo de Apoio à Saúde da Família: Aspectos legais, conceitos e possibilidades para a atuação dos profissionais de Educação Física. Palhoça: Unisul, 1ª edição, 2016.

SOCIEDADE BRASILEIRA DE CARDIOLOGIA. $7^{\text {th }}$ Brazilian Guideline of Arterial Hypertension. Chapter 6: Non-pharmacological treatment. Arquivos Brasileiros de Cardiologia, v. 107 (Suppl. 3), p. 1-83, 2016.

VAZ E, PIOVESAN, D.; FABRIS, A.V.; LINDER, J.A.; LIMA, V.; FERNANDES, D.Z. et al. Descrição do programa de avaliação e prescrição de exercícios físicos em paciente com diabetes tipo 1, depressão, síndrome metabólica e labirintite: um estudo de caso em paciente com múltiplas doenças crônicas. Anais do $11^{\circ}$ Encontro Anual de Extensão e Cultura, EAEX, Unicentro, 2018.

WARBURTON, D.E.R.; NICOL, C.W.; BREDIN, S.S.D. Health benefits of physical activity: the evidence. CMAJ, v. 174, n. 6, p. 801-809, 2006.

WASFY, M.M.; BAGGISH, A.L. Exercise dose in clinical practice. Circulation, v. 133, n. 23, p. 2297-313, 2016.

Recebido em: 13/11/2018

Aceito em: 31/07/2019 